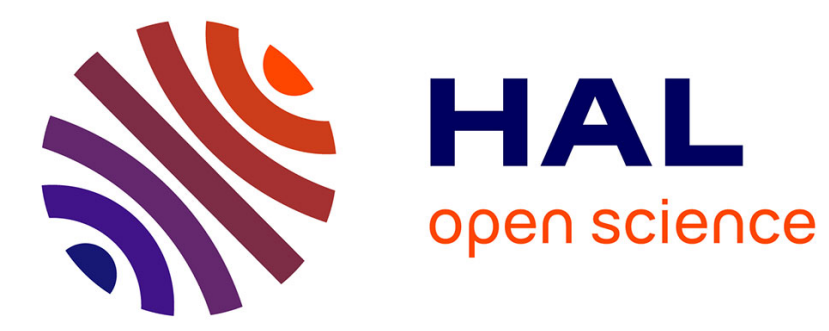

\title{
Semi-classical measures on Quantum Graphs and the Gauss map of the determinant manifold
}

Yves Colin de Verdière

\section{To cite this version:}

Yves Colin de Verdière. Semi-classical measures on Quantum Graphs and the Gauss map of the determinant manifold. Annales Henri Poincaré, 2015, 16 (2), pp.347-364. hal-00907569v2

HAL Id: hal-00907569

https://hal.science/hal-00907569v2

Submitted on 15 Feb 2014

HAL is a multi-disciplinary open access archive for the deposit and dissemination of scientific research documents, whether they are published or not. The documents may come from teaching and research institutions in France or abroad, or from public or private research centers.
L'archive ouverte pluridisciplinaire HAL, est destinée au dépôt et à la diffusion de documents scientifiques de niveau recherche, publiés ou non, émanant des établissements d'enseignement et de recherche français ou étrangers, des laboratoires publics ou privés. 


\title{
Semi-classical measures on Quantum graphs and the Gauß map of the determinant manifold
}

\author{
Yves Colin de Verdière*
}

February 15, 2014

\section{Introduction}

The so-called Quantum Ergodic Theorem (QE), due mainly to Alexander Shnirelman [Shn74, Shn93, Zel87, CdV85], asserts that the eigenfunctions of the Laplace operator on a smooth closed Riemannian manifold whose geodesic flow is ergodic are equi-distributed on the manifold in the limit of large eigenvalues provided one removes a sub-sequence of density 0. The paper [JSS13] gives QE Theorems in the case of metrics with discontinuities on smooth manifolds: the Assumptions are ergodicity of the geodesic flow which in this context is a Markov process and the fact that recombining geodesics are exceptional. The results apply also to piecewise smooth metrics on simplicial complexes, in particular to metric graphs (also called "quantum graphs"). The geodesic flow is then ergodic, but they are many recombining geodesics. Hence the second assumption is not satisfied for quantum graphs. It is currently believed that QE does not hold in general for a FIXED quantum graph. In [BKW04], it is proved that QE does not holds in the limit of large irrational star graphs. This implies that QE does not hold for a fixed large irrational star graph; this is even proved for a fixed irrational star graph. Similar results are proven in [KMW02] (put $f(x)=x^{2}$ in Equation (51)). In the literature, QE for graphs means some property of the eigenfunctions in the limit of a sequence of graphs whose number of vertices is going to infinity (see for example [GKF10]).

In the present paper, our goal is to describe all weak limits of measures $\left|\phi_{j}\right|^{2}|d x|_{\vec{l}}$, where $\left(\phi_{j}\right)$ is an orthonormal basis of eigenfunctions for the Laplacian $\Delta_{\vec{l}}$ on a metric graph $(G, \vec{l})$ and $|d x|_{\vec{l}}$ is the Riemannian measure, and to get upper bounds on the densities of the associated sub-sequences of eigenfunctions. In particular we show that $\mathrm{QE}$ does not hold for a generic metric $\vec{l}$ on a

${ }^{*}$ Université de Grenoble, Institut Fourier, Unité mixte de recherche CNRS-UJF 5582, BP 74, 38402-Saint Martin d'Hères Cedex (France); yves.colin-de-verdiere@ujf-grenoble.fr 
fixed graph, except if the graph is homeomorphic to an interval or to a circle. In order to avoid confusion, we will denote this last property by QEF (Quantum Ergodicity for Fixed graph).

If $G=(V, E)$ is a finite graph, we introduce, following ideas in [BG00, BG01], an algebraic sub-manifold $Z_{G}$, which we call the "determinant manifold", of the torus $\mathcal{T}_{E}:=(\mathbb{R} / 2 \pi \mathbb{Z})^{E}$ which allows to compute the eigenvalues thanks to the so-called "secular equation". The Gauß map $\Gamma$ associates to any point of the smooth part $Z_{G}^{\text {reg }}$ of $Z_{G}$ the half-line of the cone $\mathcal{P}_{E}:=\left[0,+\infty\left[^{E} \backslash\{0\}\right.\right.$ which is orthogonal to $Z_{G}$. Let us denote by $\mathcal{M}_{G}$ the semi-algebraic set which is the closure of the image of $\Gamma$. Let us state the main result of this paper:

Theorem 0.1 Let us fix $G$. For a generic metric $\vec{l}$, the set of non-normalized semi-classical measures is the set of all $\sum_{e \in E} m_{e}\left|d t_{e}\right|$ where $\vec{m} \in \mathcal{M}_{G}$ and where $\left|d t_{e}\right|$ is the Riemannian measure on the edge e, i.e. $t_{e}$ is an arc-length parametrization of the edge e. Moreover the densities of the corresponding eigenfunctions sub-sequences are bounded in terms of the image by $\Gamma$ of a measure $\mu_{\mathrm{BG}, \vec{l}}$ on $Z_{G}^{\mathrm{reg}}$ introduced in [BG00, BG01].

Another related result is the description of all semi-classical measures with minimal support, which are analogs of the so-called "scars" in the case of manifolds: the supports of these scars are simple paths joining two vertices of degree one and simple cycles (this is related to the paper [SK03]). From this, we deduce that QEF does not hold for a generic $\vec{l}$, except for graphs homeomorphic to intervals or circles.

Many thanks to the referees for the comments which allowed me to improve the results and the proofs.

\section{The determinant manifold of a quantum graph}

In this section, we recall the way to compute the spectra of the Laplacians $\Delta_{\vec{l}}$ on a graph $G=(V, E)$ from an algebraic hyper-surface $Z_{G}$ of the torus $\mathcal{T}_{E}=(\mathbb{R} / 2 \pi \mathbb{Z})^{E}$ which depends only on the combinatorics of the graph and not on the lengths $\vec{l}$. This approach is closely related to what people do in several papers like [BG00, BKW04, BW08, Ba12, BB13].

Let us consider a finite connected graph $G=(V, E)$ where loops and multiple edges are allowed. We choose an orientation of the edges. We associate, in the usual way, to $G$ a $1 \mathrm{D}$ singular manifold $|G|$ by gluing together \#E intervals, labelled by the set $E$, using the combinatorics of $G$. A (Riemannian) metric on $|G|$ is given by the lengths of the edges. We will denote by $\vec{l} \in] 0,+\infty\left[{ }^{E}\right.$ the collection of the lengths and the pair $(G, \vec{l})$ is called a Metric Graph.

Definition 1.1 We will say that $\vec{l}$ is irrational if the components of $\vec{l}$ are independent over the rational numbers. 
To any metric $\vec{l}$ is associated the Riemannian measure on $|G|$ denoted by $|d x|_{\vec{l}}=$ $\sum_{e \in E}\left|d t_{e}\right|$ where $t_{e} \in\left[-l_{e} / 2, l_{e} / 2\right]$ is the arc-length parametrization of the edge $e$ following the given orientation and with the origin at the center of the edge $e$. We have $\int_{|G|}|d x|_{\vec{l}}=L$ where $L=\sum_{e \in E} l_{e}$ is the total length of $(G, \vec{l})$. The Laplacian $\Delta_{\vec{l}}$ is the self-adjoint operator on $L^{2}\left(|G|,|d x|_{\vec{l}}\right)$ with domain consisting of the functions $\phi:|G| \rightarrow \mathbb{C}$ which are in the Sobolev space $\bigoplus_{e \in E} H^{2}(e)$, are continuous on $|G|$ and satisfy the Kirchhoff condition that the sums of outgoing derivatives of $\phi$ at each vertex vanish. Then $\Delta_{\vec{l}} \phi$ is given by $-d^{2} \phi / d t_{e}^{2}$ on each edge. The metric graph $G$ with the previous Laplacian is also called a Quantum graph. More generally, a quantum graph is a metric graph with a self-adjoint differential operator on $L^{2}\left(|G|,|d x|_{\vec{l}}\right)$. A good introduction to Quantum graphs is the recent book [BK13]. The operator $\Delta_{\vec{l}}$ has a discrete spectrum given by $\lambda_{j}=k_{j}^{2}$ with $k_{1}=0<k_{2} \leq \cdots$. We denote by $\phi_{j}:|G| \rightarrow \mathbb{C}$ an associated orthonormal basis of eigenfunctions. Let us remark that we can assume that $G$ has no vertex of degree 2 , because we allow multiple edges and loops.

Let us try to compute the non-zero part of the spectrum of $\Delta_{\vec{l}}$ : let us look for an eigenfunction $\phi$ with eigenvalue $k^{2}$ with $k>0$. On the edge $e$, we have

$$
\phi\left(t_{e}\right)=a_{e} \cos k t_{e}+b_{e} \sin k t_{e} .
$$

Let us define $\xi_{e}=\cos k l_{e} / 2$ and $\eta_{e}=\sin k l_{e} / 2$. From the continuity conditions and the Kirchhoff conditions, we get, for the function $\phi=\phi_{a, b}$, a linear system

$$
L_{\xi, \eta}(a, b)=0
$$

of $2 \# E$ equations with the same number of unknowns, because the values of the function $\phi$ and its derivatives divided by $k$ at the vertices are (linear) functions of $\xi_{e}$ and $\eta_{e}$. Let us remark that if we change any $\left(\xi_{e}, \eta_{e}\right)$ into $\left(-\xi_{e},-\eta_{e}\right)$, the solutions of the new system are changed by $\left(a_{e}, b_{e}\right) \rightarrow\left(-a_{e},-b_{e}\right)$.

The determinant of the system $L_{\xi, \eta}(a, b)=0$ is an homogeneous polynomial of degree 2 with respect to each $\zeta_{e}=\left(\xi_{e}, \eta_{e}\right)$. Hence, it can be expressed as a polynomial of total degree \#E in the variables $z_{e}=\left(x_{e}, y_{e}\right)$ with $x_{e}=\cos k l_{e}, y_{e}=$ $\sin k l_{e}$ which is of degree 1 w.r. to each pair $\left(x_{e}, y_{e}\right)$. Let $\theta_{e}$ be the corresponding angles so that $x_{e}=\cos \theta_{e}, y_{e}=\sin \theta_{e}$. Let us denote this trigonometric polynomial $\delta_{G}(\theta)$ viewed as a function on the torus $\mathcal{T}_{E}=(\mathbb{R} / 2 \pi \mathbb{Z})^{E}$ whose coordinates are denoted by $\theta=\left(\theta_{e}\right)_{e \in E}$. The following fact is clear from what we said, the number $k^{2}, k>0$ is an eigenvalue of $\Delta_{\vec{l}}$ if and only if $\delta_{G}([k \vec{l}])=0$ where [ ] means that we take the components modulo $2 \pi$. In other words, $[k \vec{l}]$ belongs to the algebraic hyper-surface $Z_{G}$ of equation $\delta_{G}=0$ of $\mathcal{T}_{E}$. Let us remark that, by an elementary check, $\theta=0$ belongs too to $Z_{G}$.

Let us discuss a few elementary property of $Z_{G}$. We will say that $[k \vec{l}]$ is a smooth point of $Z_{G}$ if the differential of $\delta_{G}$ at that point does not vanish. A point which is not smooth is called singular. 
Theorem 1.1 If $k^{2}>0$ is a non degenerate eigenvalue of $\Delta_{\vec{l}}$, the point $[k \vec{l}]$ is a smooth point of $Z_{G}$ and the tangent space to $Z_{G}$ at that point is given by $\sum_{e} m_{e} d \theta_{e}=0$ where $m_{e}=a_{e}^{2}+b_{e}^{2}$ for an eigenfunction given by Equation (1).

Conversely, if the eigenvalue $k^{2}$ of $\Delta_{\vec{l}}$ is degenerate, $[k \vec{l}]$ is a singular point of $Z_{G}$.

The manifold $Z_{G}$ is singular in a set of co-dimension at least 1 (w.r. to $Z_{G}$ itself) except if $|G|$ is a circle.

Let us denote by $Z_{G}=Z_{G}^{\mathrm{reg}} \cup Z_{G}^{\text {sing }}$ the partition of $Z_{G}$ into the sets of regular and singular points. Using a result of L. Friedlander on the genericity of non degeneracy of the eigenvalues of $\Delta_{\vec{l}}$ [Fr05] if $|G|$ is not a circle, we get the fact that, if $|G|$ is not a circle, the set $Z_{G}^{\text {reg }}$ is dense in $Z_{G}$ and hence, because $Z_{G}$ is an algebraic manifold, the set of singular points $Z_{G}^{\text {sing }}$ has co-dimension larger than one w.r. to $Z_{G}$.

Questions: as we will see from the examples in Sections 6.2 and $6.3, Z_{G}$ can be reducible. For which graphs is $Z_{G}$ irreducible? A conjecture could be that $Z_{G}$ is reducible, if and only if $|G|$ admits a non trivial symmetry group which is an isometry group for each metric and $|G|$ is not an interval, more precisely:

Proposition 1.1 The topological set $|G|$ admits a non trivial symmetry group which acts by isometry for each choice of a metric $\vec{l}$ if and only if either $|G|$ is homeomorphic to $[-1,1]$ (then the isometry group is generated by $x \rightarrow-x$ ), either $\mid G-i s$ a circle with the antipody as a non trivial symmetry, or if $|G|$ is obtained by adding to a $|K|$ (associated to a graph $K$ ) some loops (at least 1) attached to vertices of $K$ (then the symmetry group is generated by the involutions $x \rightarrow-x$ on one of the loops parametrized by $-1 \leq x \leq+1$ ).

Proof.-

We can assume that $G$ has no vertex of degree 2. If $G$ is a circle or an interval it admits clearly such a symmetry group. Let us assume moreover that $G$ has no loops as in the Proposition. Then $G$ admits a vertex of degree at least 3 . Because the lengths of these edge are arbitrary, the isometries have to fix this vertex and the adjacent adges. An easy induction gives the result.

If the manifold $Z_{G}$ is irreducible, is the set of singular point of co-dimension $\geq 2$ w.r. to $Z_{G}$ as expected from the generic co-dimension of symmetric matrices with som degenerate eigenvalue?

Proof.-

(of Theorem 1.1) 
- Using the homogeneity of the spectrum, i.e. , $\forall c>0, k_{j}(c \vec{l})=$ $c^{-1} k_{j}(\vec{l})$, we can assume that $k=1$. In other words $Z_{G}$ is identified with the $[\vec{l}]$ 's so that 1 is an eigenvalue of $\Delta_{\vec{l}}$. Let $z_{0}=[\vec{l}] \in Z_{G}$. We assume that the eigenvalue 1 is non-degenerate for $\Delta_{\vec{l}}$. Close to $z_{0}$, the set $Z_{G}$ is defined by the equation $k([\vec{l}])=1$ meaning that 1 is an eigenvalue of $\Delta_{\vec{l}}$, so that $Z_{G}$ is smooth if $d k \neq 0$ and the tangent space is the kernel of $d k$. From the calculation of $d k$ in Appendix A, we get the result.

- Let us assume that $k_{0}^{2}>0$ is a degenerate eigenvalue of $\Delta_{\vec{l}}$. With the notations around Equation (1), this implies that dim ker $L_{\xi_{0}, \eta_{0}} \geq$ 2 with $\xi_{0}=\left(\cos k_{0} l_{e}\right), \eta_{0}=\left(\sin k_{0} l_{e}\right)$. This implies that the diffenrential of the determinant of $L_{\xi_{0}, \eta_{0}}$ with respect to any variation of $\xi, \eta$ vanishes. The result follows.

The singularities of $Z_{G}$ are associated to the degenerate eigenvalues. A description of these singularities would be of interest, already for general star graphs or complete graphs. The case of diabolical singularities, corresponding to eigenvalues of multiplicity two, is used in [BG00] in order to study the behavior as $s \rightarrow 0$ of the distribution $P(s) d s$ of the level spacing $\lambda_{j+1}-\lambda_{j}$ defined by

$$
P(s) d s=\lim _{\lambda \rightarrow \infty} \frac{\#\left\{j \mid s \leq \lambda_{j+1}-\lambda_{j} \leq s+d s\right\}}{\#\left\{j \mid \lambda_{j} \leq \lambda\right\}} .
$$

\section{The Gauß map and semi-classical measures}

Let us denote by $\mathcal{P}_{E}$ the cone $\left[0,+\infty\left[{ }^{E} \backslash\{0\}\right.\right.$. For any $\vec{m}=\left(m_{e}\right)_{e \in E} \in \mathcal{P}_{E}$ and any $\vec{l}$, we identify $\vec{m}$ with the measure $\sum_{e \in E} m_{e}\left|d t_{e}\right|$ on $|G|$. In other words, if $\vec{l}$ is given, we consider $\mathcal{P}_{E}$ as a sub-cone of the cone of positive Radon measures on $|G|$. Our goal is to characterize the measures which are semi-classical limits. We will need a

Definition 2.1 If $\phi$ is an eigenfunction of some $\Delta_{\vec{l}}$ with eigenvalue $k^{2}>0$, the restriction of $\phi$ to the edge e writes $\phi\left(t_{e}\right)=a_{e} \cos k t_{e}+b_{e} \sin k t_{e}$; we denote by $\mu_{\phi}$ the measure on $|G|$ defined by $\mu_{\phi}=\sum_{e}\left(a_{e}^{2}+b_{e}^{2}\right)\left|d t_{e}\right|$ which belongs to $\mathcal{P}_{E}$.

Definition 2.2 A semi-classical limit for $\Delta_{\vec{l}}$ is a weak limit of a sequence of measures $\sum_{e \in E}\left|\phi_{k_{j}}\left(t_{e}\right)\right|^{2}\left|d t_{e}\right|$ on $|G|$ where $\phi_{k_{j}}$ are non-zero eigenfunctions of $\Delta_{\vec{l}}$ with eigenvalues $k_{j}^{2} \rightarrow+\infty$.

Remark 2.1 We do not use a lift of the measures to the cotangent space as in the Shnirelman Theorem. 
Remark 2.2 We do not ask the eigenfunctions to be $L^{2}$-normalized. Our semiclassical limits are in general not probability measures.

We can summarize the results as follows:

Theorem 2.1 1. Let $\mu=\sum_{e \in E} m_{e}|d t|_{e}$ be a semi-classical limit for $\Delta_{\vec{l}_{0}}$, then there exists $\overrightarrow{l_{\infty}}$ and an eigenfunction $\phi_{\infty}$ of $\Delta_{\vec{l}_{\infty}}$ associated to the eigenvalue 1 so that $\mu=\mu_{\phi_{\infty}}$.

2. Let us assume that $\phi$ is an eigenfunction of some $\Delta_{\overrightarrow{l_{0}}}$ with a simple eigenvalue $k_{0}^{2}$, then $\mu_{\phi}$ is a semi-classical measure for $\Delta_{\overrightarrow{l_{0}}}$ and for any $\Delta_{\vec{l}}$ with $\vec{l}$ irrational.

3. Let $\mathcal{G}_{G}$ be the set of the $\vec{l}$ so that $\vec{l}$ is irrational and the line $\{[k \vec{l}] \mid k>0\}$ does not meet $Z_{G}^{\text {sing }}$ (or equivalently the set of $\vec{l}$ so that the eigenvalues of $\Delta_{\vec{l}}$ are simple). If $|G|$ is not a circel, the set $\mathcal{G}_{G}$ is Baire generic and, if $\overrightarrow{l_{0}} \in \mathcal{G}_{G}$, the set of semi-classical measures for $\Delta_{\vec{l}_{0}}$ is the closure of the image of $Z_{G}^{\mathrm{reg}}$ by the Gauß map.

Proof.-

1. Let us consider a sequence of eigenfunctions of $\Delta_{\vec{l}}$ defined by $\phi_{k_{j}}\left(t_{e}\right)=a_{e, j} \cos k_{j} t_{e}+b_{e, j} \sin k_{j} t_{e}$. Then, for large $k_{j}$ 's, the measures $\sum_{e} \phi_{k_{j}}\left(t_{e}\right)^{2}\left|d t_{e}\right|$ have a weak limit if and only if the limits $m_{e}=\lim _{j \rightarrow \infty}\left(a_{e, j}^{2}+b_{e, j}^{2}\right)$ exist and this limit measure is given by $\sum_{e \in E} m_{e}|d t|_{e}$. By compactness of $Z_{G}$, we can assume that $\left[k_{j} \overrightarrow{l_{0}}\right]$ converges to $\left[\overrightarrow{l_{\infty}}\right]$ and we can assume that all components of $\overrightarrow{l_{\infty}}$ are $>0$. Then we can again extract a sub-sequence so that the numbers $a_{e, j}$ and $b_{e, j}$ converge to some limit which will be associated, by the closeness of the eigenfunctions equations, to an eigenfunction of $\Delta_{l_{\infty}}$ with eigenvalue 1 .

2. From the assumption and Theorem 1.1, we get that the set $Z_{G}$ is smooth near $\left[k_{0} \overrightarrow{l_{0}}\right]$ and transverse to any curve $t \rightarrow[t \vec{l}]$ if the components of $\vec{l}$ are all $>0$. From this and the fact that the linear flows on tori are recurrent, we can get a sequence $k_{j} \rightarrow+\infty$ so that $\left[k_{j} \overrightarrow{l_{0}}\right] \rightarrow\left[k_{0} \overrightarrow{l_{0}}\right]$ as $j \rightarrow \infty$ and we have that $\mu_{\phi_{k_{j}}} \rightarrow \mu_{\phi}$. Similarly, if $\vec{l}$ is irrational, we can find $k_{j} \rightarrow \infty$ so that $\left[k_{j} \vec{l}\right] \in Z_{G}$ and $\left[k_{j} \vec{l}\right] \rightarrow\left[k_{0} \overrightarrow{l_{0}}\right]$.

3. The genericity comes from the fact that $Z_{G}^{\text {sing }}$ is of co-dimension at least two in $\mathcal{T}_{E}$. From the previous part, we know already that each measure in the image of the Gauß map is a semi-classical 
measure and the same is true for a measure in the closure of this image, because the set of semi-classical measures is closed. On the other hand, we know that each semi-classical measure is the limit of a sequence of $\mu_{\phi_{k_{j}}}$; because of the assumption on $\vec{l}$ this implies that each semi-classical measure lies in the closure of the image of the Gauß map.

\section{The Barra-Gaspard measure, Weyl formula and the densities of semi-classical limits}

In [BG00], the authors introduce a $\vec{l}$-dependent measure on $Z_{G}^{\mathrm{reg}}$, which we will denote by $\mu_{\mathrm{BG}, \vec{l}}$. The manifold $Z_{G}^{\mathrm{reg}}$ is transverse to any vector with strictly positive coordinates and hence oriented. The measure $\mu_{\mathrm{BG}, \vec{l}}$ is defined ${ }^{1}$ by

$$
\mu_{\mathrm{BG}, \vec{l}}=(2 \pi)^{-\# E}\left|\iota(\vec{l}) \wedge_{e \in E} d \theta_{e}\right|
$$

$(\iota(\vec{V}) \omega$ is the inner product), which is a Radon measure with strictly positive density everywhere on $Z_{G}^{\mathrm{reg}}$.

Proposition 3.1 The mass of the measure $\mu_{\mathrm{BG}, \vec{l}}$ is exactly $L / \pi$.

Proof.-

The total mass of the measure $\mu_{\mathrm{BG}, \vec{l}}$ is less than $L / \pi$ : let us consider, for $e \in E$, the canonical projection $\pi_{e}: Z_{G} \rightarrow(\mathbb{R} / 2 \pi \mathbb{Z})^{E \backslash e}$. From the definition of $\mu_{\mathrm{BG}, \vec{l}}$, we get

$$
\int_{Z_{G}} \mu_{\mathrm{BG}, \vec{l}}=\sum_{e \in E} l_{e} \int_{(\mathbb{R} / 2 \pi \mathbb{Z})^{E \backslash e}} \operatorname{deg}_{e}(\theta)\left|\wedge{\widehat{d \theta_{e}}}\right|
$$

where $\operatorname{deg}_{e}(\theta)$ is the cardinal of $\pi_{e}^{-1}(\theta)$. The fact that $\delta_{G}$ is of degree 1 w.r. to each $\left(\cos \theta_{e}, \sin \theta_{e}\right)$ implies that $\operatorname{deg}_{e} \leq 2$ and the upper bound of the mass.

In fact $\operatorname{deg}_{e} \equiv 2$ as follows from Lemma 3.2 below and the Weyl asymptotic formula (see [BK13] p. 95)

\footnotetext{
${ }^{1}$ Avoiding the intrinsic calculus of exterior differential forms, the Barra-Gaspard measure can be defined in the following less "cryptic" way: if $d \sigma_{Z}$ is the measure on $Z_{G}^{\text {reg }}$ associated to the metric induced by the flat metric $\sum_{e \in E} d \theta_{e}^{2}$ on $\mathcal{T}_{E}$ and $\pm \vec{\nu}$ are the unit normal vector fields to $Z_{G}^{\text {reg }}$, we put

$$
\mu_{\mathrm{BG}, \vec{l}}:=(2 \pi)^{-\# E}|\vec{\nu} . \vec{l}| d \sigma_{Z} .
$$
}


Theorem 3.1 We have (Weyl's law)

$$
\lim _{K \rightarrow \infty} \frac{1}{K} \#\left\{0 \leq k \leq K \mid[k \vec{l}] \in Z_{G}\right\}=\frac{L}{\pi} .
$$

We will need the following Lemma, similar to Proposition 4.4 of [BW08] and coming also from the ideas of Barra-Gaspard:

Lemma 3.1 If $\vec{l}$ is irrational and $D \subset Z_{G}^{\mathrm{reg}}$ is a compact domain with piecewise smooth boundary, we have

$$
\lim _{K \rightarrow \infty} \frac{1}{K} \#\left\{k_{j} \mid 0<k_{j} \leq K,\left[k_{j} \vec{l}\right] \in D\right\}=\int_{D} \mu_{\mathrm{BG}, \vec{l}} .
$$

Similarly, if $f: Z_{G} \rightarrow \mathbb{R}$ has a compact support in $Z_{G}^{\mathrm{reg}}$, we have:

$$
\lim _{K \rightarrow \infty} \frac{1}{K} \sum_{0<k_{j} \leq K,\left[k_{j} \vec{l}\right] \in Z_{G}} f\left(\left[k_{j} \vec{l}\right]\right)=\int_{Z_{G}} f \mu_{\mathrm{BG}, \vec{l}} .
$$

Proof.-

Let us choose $\varepsilon>0$ small enough so that the map $F:[-\varepsilon, \varepsilon] \times D \rightarrow$ $\mathcal{T}_{E}$, defined by $F(t, \theta)=\theta+t \vec{l}$, is a smooth embedding of image $D_{\varepsilon}$. From the unique ergodicity of the Kronecker flows on the tori, we get

$$
\lim _{K \rightarrow \infty} \frac{1}{K}\left|\left\{k \mid 0<k \leq K,[k \vec{l}] \in D_{\varepsilon}\right\}\right|=(2 \pi)^{-\# E} \operatorname{vol}\left(D_{\varepsilon}\right)
$$

where $|X|$ denote the Lebesgue measure of a set $X \subset \mathbb{R}$. We observe that $[k \vec{l}]$ belongs to $D_{\varepsilon}$ if and only if there exists $j$ so that $\left[k_{j} \vec{l}\right] \in D$ and $k \vec{l}=\left[k_{j} \vec{l}\right]+t \vec{l}$ with $|t| \leq \varepsilon$. The lefthandside of Equation (2) is equal to

$$
\lim _{K \rightarrow \infty} \frac{2 \varepsilon}{K} \#\left\{k_{j} \mid 0<k_{j} \leq K,\left[k_{j} \vec{l}\right] \in D\right\}
$$

while, using the definition of $\mu_{\mathrm{BG}, \vec{l}}$, the righthandside of Equation (2) is equal to $2 \varepsilon \mu_{\mathrm{BG}, \vec{l}}(D)$. follows:

We can remove the Assumption that $D$ is compactly supported in $Z_{G}^{\text {reg }}$ as

Lemma 3.2 If $\vec{l}$ belongs to $\mathcal{G}_{G}$, we have

$$
\lim _{K \rightarrow \infty} \frac{1}{K} \#\left\{k_{j} \mid 0<k_{j} \leq K,\left[k_{j} \vec{l}\right] \in Z_{G}\right\}=\int_{Z_{G}} \mu_{\mathrm{BG}, \vec{l}} .
$$

A similar result holds for the integration of a continuous function $f$ on $Z_{G}$ :

$$
\lim _{K \rightarrow \infty} \frac{1}{K} \sum_{0<k_{j} \leq K,\left[k_{j} \vec{l}\right] \in Z_{G}} f\left(\left[k_{j} \vec{l}\right]\right)=\int_{Z_{G}} f \mu_{\mathrm{BG}, \vec{l}} .
$$


Proof.-

Let $R_{\varepsilon}$ be the ball of radius $\varepsilon$ in $Z_{G}$ centered on $Z_{G}^{\text {sing }}$ and $\tilde{R}_{\varepsilon}$ be the set

$$
\tilde{R}_{\varepsilon}:=\left\{z+t \vec{l} \mid z \in R_{\varepsilon}, 0 \leq t \leq \varepsilon\right\} .
$$

We have $\operatorname{vol}\left(\tilde{R}_{\varepsilon}\right)=O\left(\varepsilon^{2}\right)$, because the codimension of $Z_{G}^{\text {sing }}$ in $\mathcal{T}_{E}$ is larger than 2. Using the result of Appendix D, there exists a constant $C>0$, so that, for any $n \in \mathbb{N}$, we have:

$\#\left\{k \mid n<k \leq n+1,[k \vec{l}] \in R_{\varepsilon}\right\} \leq \frac{C}{\varepsilon}\left|\left\{k^{\prime} \mid n-\varepsilon \leq k^{\prime} \leq n+1+\varepsilon,\left[k^{\prime} \vec{l}\right] \in \tilde{R}_{\varepsilon}\right\}\right|$

(if $\left[k^{\prime} \vec{l}\right]$ belongs to $\tilde{R}_{\varepsilon}$, there exists $k$ so that $[k \vec{l}] \in R_{\varepsilon}$ and $\left|k-k^{\prime}\right| \leq \varepsilon$; the constant $C$ is an uniform upper bound on the number of solutions of $\delta_{G}(k \vec{l})=0$ with $\left.n<k \leq n+1\right)$. Assuming that $K$ is an integer and summing the previous inequalities from $n=0$ to $n=K-1$, we get a constant $\tilde{C}$ so that

$\frac{1}{K} \#\left\{k \mid 0<k \leq K,[k \vec{l}] \in R_{\varepsilon}\right\} \leq \frac{\tilde{C}}{\varepsilon K}\left|\left\{k^{\prime} \mid 0 \leq k^{\prime} \leq K+1,\left[k^{\prime} \vec{l}\right] \in \tilde{R}_{\varepsilon}\right\}\right|$.

The limit of the righthandside as $K \rightarrow \infty$ is $\tilde{C} / \varepsilon$ times the volume of $\tilde{R}_{\varepsilon}$ and can be close to 0 by choosing $\varepsilon$ small enough. The proof is completed by writing $Z_{G}=\left(Z_{G} \cap R_{\varepsilon}\right) \cup\left(Z_{G} \backslash Z_{G} \cap R_{\varepsilon}\right)$, by using Lemma 3.1 for the main term and by using the finitness of the mass of $\mu_{\mathrm{BG}, \vec{l}}$ (first part of the proof of Proposition 3.1).

The Barra-Gaspard measure is related to the densities of sequences of eigenvalues giving a given semi-classical limit. This density vanishes in general, so we have to say it in the following way:

Theorem 3.2 Let $D \subset \mathcal{P}_{E}$ be a compact domain with smooth boundary and consider a sequence of eigenfunctions $\phi_{k_{j}}$ of some $\Delta_{\vec{l}}$ with $\vec{l}$ generic. Let us assume that all semi-classical limits of the $\mu_{\phi_{k_{j}}}$ lie in $D$, then the density of the sequence $k_{j}$ is bounded from above in terms of $\mu_{\mathrm{BG}, \vec{l}}$ as follows:

$$
\limsup _{K \rightarrow \infty} \frac{\#\left\{k_{j} \leq K\right\}}{K} \leq \mu_{\mathrm{BG}, \vec{l}}\left(\Gamma^{-1}(D)\right)
$$

The converse is true; for any generic $\vec{l}$ and for any compact domain $D \subset \mathcal{P}_{E}$, there exists a sub-sequence $\phi_{k_{j}}$ of density $\mu_{\mathrm{BG}, \vec{l}}\left(\Gamma^{-1}(D)\right)$ so that the semi-classical limits associated to sub-sequences of $\phi_{k_{j}}$ belongs to $D$.

Proof.- 
Let $D^{\prime} \subset \mathcal{P}_{E}$ a neighborhood of $D$. Then for $j$ large enough, $\Gamma_{\vec{l}}\left(\left[k_{j} \vec{l}\right]\right)$ belongs to $D^{\prime}$. So that, from a slight extension of Lemma 3.2 to domains in $Z_{G}$, we get the upper bound $\mu_{\mathrm{BG}, \vec{l}}\left(\Gamma_{\vec{l}}^{-1}\left(D^{\prime}\right)\right.$.

For, the converse, it is enough to take the sequence of $k_{j}$ 's so that $\left[k_{j} \vec{l}\right]$ belongs to $\Gamma^{-1}(D)$.

We get the following

Corollary 3.1 If $\vec{l}$ belongs to $Z_{G}^{\mathrm{reg}}$ and $\vec{l}$ is generic, let $\phi$ be an eigenfunction of $\Delta_{\vec{l}}$ with eigenvalue 1 . Then, for all neighborhoods $U$ of $\mu_{\phi}$, there exists sub-sequences of positive densities of the eigenfunctions of $\Delta_{\vec{l}}$ so that the semi-classical limits of sub-sequences belongs to $\Gamma(U)$.

Corollary 3.2 If $\vec{l}$ is generic and there exists an eigenfunction $\phi$ of $\Delta_{\vec{l}}$ so that $\mu_{\phi}$ is not the Liouville measure, then QEF does not holds for $(G, \vec{l})$.

Moreover, we have the following link with the Liouville measure:

Theorem 3.3 Let $\Gamma_{\vec{l}}$ be the map $\Gamma$ normalized so that the sums $\sum m_{e}\left|d t_{e}\right|$ are probabilities, then

$$
\int \Gamma_{\vec{l}}(\theta) d \mu_{\mathrm{BG}, \vec{l}}=\frac{1}{\pi}\left(\sum_{e}\left|d t_{e}\right|\right) .
$$

This can be reformulated as "The average of the Gauß map with respect to $\mu_{\mathrm{BG}, \vec{l}}$ is the Liouville measure".

Proof.-

Le us define

$$
A_{e}(K)=\frac{l_{e}}{2 K} \sum_{0 \leq k_{j} \leq K}\left(a_{j, e}^{2}+b_{j, e}^{2}\right)
$$

where the normalized eigenfunction $\phi_{j}$ writes, on the edge $e, \phi_{j}\left(t_{e}\right)=$ $a_{j, e} \cos k t_{e}+b_{j, e} \sin k t_{e}$. From the local Weyl Theorem and the fact that

$$
\int_{e}\left|\phi_{j}\left(t_{e}\right)\right|^{2} d t_{e}=\frac{l_{e}}{2}\left(a_{j, e}^{2}+b_{j, e}^{2}\right)+O(1 / k)
$$

we get that

$$
\lim _{K \rightarrow \infty} A_{e}(K)=l_{e} / \pi .
$$

On the other hand, we have

$$
\Gamma_{\vec{l}}\left(\left[k_{j} \vec{l}\right]\right)=\frac{1}{2}\left(a_{j, e}^{2}+b_{j, e}^{2}\right)+O(1 / k) .
$$

Using Lemma 3.2, we get

$$
\lim _{K \rightarrow \infty} A_{e}(K)=l_{e} \int_{Z_{G}}\left(\Gamma_{\vec{l}}(\theta)\right)_{e} d \mu_{\mathrm{BG}, \vec{l}} .
$$




\section{Scars}

Let us look at semi-classical measures with small supports; we have the:

Theorem 4.1 Let $G$ be given. The minimal supports of the semi-classical measures for a generic $\vec{l}$ are the simple cycles and the simple paths between two vertices of degree 1 of $G$. These measures are extremal points of the convex hull of all semi-classical measures.

We start with the

Lemma 4.1 Let $K \subset|G|$ be the support of a semi-classical measure $\mu$. Then $K$ is an union of edges of $G$ and every vertex of $K$ is of degree $\geq 2$ in $K$ or is of degree 1 in $G$.

Proof of the Lemma.-

From the first Assertion of Theorem 2.1, we deduce that $\mu=\mu_{\phi}$ where $\phi$ is an eigenfunction of some $\Delta_{l_{\infty}}$ on $G$. The Lemma follows then from the Kirchoff conditions applied to $\phi$.

Proof of the Theorem.-

- Simple paths joining to vertices of degree 1 are minimal supports: Let $\gamma$ be a simple oriented path whose $N$ vertices are $(1,2, \cdots, N)$ and choose a vector $\vec{l}$ so that the lengths of the edges of the path are $\vec{l}_{0}=(1 / 2,1,1, \cdots, 1,1 / 2)$. If we parametrize this path by $0 \leq t \leq N-2$, the function $\phi_{1}$ defined by $\phi_{1}=\cos t$ on $\gamma$ and 0 outside is an eigenfunction of $\Delta_{\vec{l}}$ with eigenvalue $\pi^{2}$. I claim that we can choose such a vector $\vec{l}$ extending $\vec{l}_{0}$ so that the eigenvalue $\pi^{2}$ of $\Delta_{\vec{l}}$ is simple: if it is not, let $X:=\operatorname{ker}\left(\Delta_{\vec{l}}-\pi^{2}\right)$, and $l_{t}$ defined by $\left(l_{t}\right)_{e}=(1-t) l_{e}$ for $e \notin \gamma$ and $\left(l_{t}\right)_{e}=l_{e}$ for $e \in \gamma$. Then the quadratic form on $X$ defined by $f \rightarrow\langle\dot{\Delta} f \mid f\rangle$ vanishes on $\phi_{1}$ and is $>0$ in any other direction (because as we have seen the support of $\phi_{1}$ is minimal). It implies that $\pi^{2}$ is a simple eigenvalue for $\Delta_{\overrightarrow{l_{t}}}$ for small non-zero $t$. It follows that the uniform measure on $\gamma$ is a semi-classical measure for this choice of $\vec{l}$ and hence for all generic $\vec{l}$ 's.

The minimality follows from Lemma 4.1.

- Simple cycles are minimal supports: Let $\gamma$ be a simple oriented cycle whose $N$ vertices are $(1,2, \cdots, N-1, N=1)$ and choose a vector $\vec{l}$ so that the lengths of the edges of $\gamma$ are 1 . By an 
argument similar to the case of a simple path, we get that the uniform measure on $\gamma$ is a semi-classical measure for this choice of $\vec{l}$ and all irrational $\vec{l}$ 's.

Again the minimality follows from lemma 4.1.

- If $K$ is the support of a semi-classical measure, $K$ contains a simple path joining 2 vertices of degree 1 of $G$ or a simple cycle of $G$ : if $K$ contains no simple cycles, $K$ is a forest and every vertex of degree 1 of $K$ is also of degree 1 in $G$. So that every sub-tree of $K$ contains simple paths joining 2 vertices of degree 1 in $G$.

As a Corollary, we get

Theorem 4.2 For a generic $\vec{l}$, QEF holds for $\Delta_{\vec{l}}$ if and only if $|G|$ is homeomorphic to a circle or to an interval.

Any connected graph which is not homeomorphic to a circle or an interval contains as strict sub-graphs a simple cycle or a path whose ends are of degree one. The Theorem 3.3 implies that the image of $Z_{G}^{\text {reg }}$ by the Gauß map contains at least one line distinct from the Liouville measure. The proof is completed by applying Corollary 3.2.

\section{$5 \quad$ Ergodicity of the geodesic flow on graphs}

In the case of a connected quantum graph, the classical dynamics is ergodic as shown from the study of the associated Perron-Frobenius operator done in [BG01]. The phase space $Z$ of a Quantum graph $G=(V, E, \vec{l})$ (the unit cotangent bundle) can be identified with the set of oriented edges: to a point $x$ of an oriented edge, we associate the unit co-vector pointing in the direction given from the orientation. Using the probabilities given from the transition coefficients in Appendix B, we get the geodesic flow on $Z$ as a Markov process. We have the

Theorem 5.1 If $G$ is connected and is not homeomorphic to a circle, the geodesic flow on $(V, E, \vec{l})$ is ergodic.

In order to prove the previous result, we introduce the Perron-Frobenius semigroup $T_{t}, t \geq 0$ acting on $L^{\infty}\left(|G|,|d x|_{\vec{l}}\right)$ as follows:

$$
T_{t} f(x)=\sum_{\gamma:[0, t] \rightarrow|G|, \gamma(0)=x} w(\gamma) f(\gamma(t)),
$$

where the sum is over the continuous paths with speed 1 starting from $x$ at time 0 and $w(\gamma)$ is the product of the transitions probabilities at all $t$ for which $\gamma(t)$ is a vertex.

Proof.- 
It is enough to prove that the only functions invariant by the PerronFrobenius semi-group $\left(T_{t}\right)_{t>0}$ are the constant functions. We see first that such a function $f$ has to be constant on each oriented edge by using at a point $x$ the invariance by $T_{t}$ for $t$ small. Moreover if $G$ is connected and is not homeomorphic to a circle, the geodesic flow is transitive: for any pair $x, y \in Z$ there exists a geodesic from $x$ to $y$. Let us choose $f$ constant on the oriented edges and invariant by the semi-group $\left(T_{t}\right)_{t>0}$. Let us choose an edge $e_{0}$ sot that $\forall x \in$ $Z, f(x) \leq f\left(e_{0}\right)$ and $x_{0} \in e_{0}$. We have $T_{t} f\left(x_{0}\right)=f\left(x_{0}\right)$ and $T_{t} f\left(x_{0}\right)=$ $\sum_{\gamma:[0, t] \rightarrow Z} w(\gamma) f(\gamma(t))$. Using the fact that $\sum_{\gamma:[0, t] \rightarrow Z} w(\gamma)=1$, we get that $f(y)=f\left(x_{0}\right)$ if there is a geodesic $\gamma$ so that $\gamma(0)=x_{0}$ and $\gamma(t)=y$. This holds for all $y \in Z$ if the geodesic flow is transitive.

From the previous study, we see that the QEF Theorem is not valid for Quantum Graphs even if the geodesic flow is ergodic, which is the case for all connected graphs which are not homeomorphic to a circle. In view of the main result of [JSS13] which says that QE holds if the geodesic flow is ergodic and the set of recombining geodesics is of measure 0 , this is due to the fact that there are a lot of recombining geodesics: for example in a star graph, they are many geodesics starting from $x$ in an edge $e_{1}$ an coming back to $x$ in the opposite direction following a geodesic containing $k_{1}$ times the edge $e_{1}$ and $k_{2}$ times the edge $e_{2}$ with $k_{1}, k_{2} \geq 1$ and with different orders.

\section{Examples}

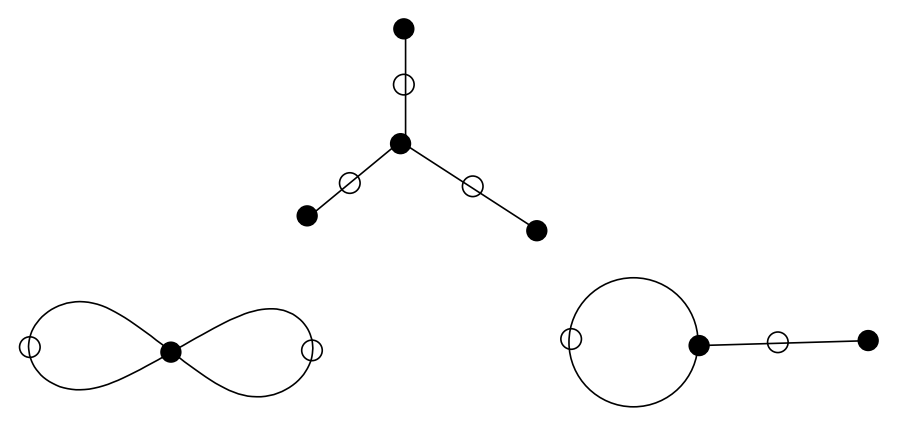

Figure 1: The 3 examples: the black circles are the vertices and the white circles are the middle of the edges 


\subsection{The star graph with three edges}

Let us consider for simplicity the case of a star graph with three edges $G=(V, E)$ where $l_{1}, l_{2}, l_{3}$ are the lengths of three edges. The function $\delta_{G}$ can be computed as follows: we assume that, we parametrize the $j$ th edge by $0 \leq t_{j} \leq l_{j}$ where 0 corresponds to the vertex of degree 1 . Then an eigenfunction of eigenvalue $k^{2}$ will be of the form $\phi\left(t_{j}\right)=a_{j} \cos k t_{j}$. Putting $x_{j}=\cos k t_{j}$ and $y_{j}=\sin k t_{j}$, we get the three equations

$$
a_{1} x_{1}=a_{2} x_{2}=a_{3} x_{3}, a_{1} y_{1}+a_{2} y_{2}+a_{3} y_{3}=0 .
$$

Hence

$$
\delta_{G}(x, y)=x_{1} x_{2} y_{3}+x_{2} x_{3} y_{1}+x_{3} x_{1} y_{2} .
$$

Singularities of $\Sigma$ : The surface $\Sigma_{G} \subset \mathcal{T}_{E}$ is invariant by the eight translations whose vectors have coordinates 0 or $\pi$. We will restrict ourselves to the intersection of $\Sigma_{G}$ with the cube $C=[-\pi / 2, \pi / 2]^{3}$. The seven other cubes are deduced by translations. The surface $\Sigma_{G}$ is also invariant by the central symmetry $\sigma:\left(\theta_{j}\right) \rightarrow\left(-\theta_{j}\right)$.

We can cover $\Sigma$ by four types of charts and use adapted coordinates taken from the set $X_{j}=x_{j} / y_{j}, Y_{j}=y_{j} / x_{j}$ :

1. In the domain $x_{1} x_{2} x_{3} \neq 0$, we get $Y_{1}+Y_{2}+Y_{3}=0$ which is smooth.

2. In the domain $y_{1} y_{2} y_{3} \neq 0$, we get $X_{1} X_{2}+X_{2} X_{3}+X_{3} X_{1}=0$ which is singular at the eight points where all $x_{j}$ vanish. They are "diabolical" points located at the vertices of the cubes.

3. In the domains, $x_{1} x_{2} y_{3} \neq 0$ and $x_{1} y_{2} y_{3} \neq 0, \Sigma_{G}$ is smooth.

The boundary of the closure of the intersection $\left.\Sigma_{0}=\Sigma \cap\right]-\pi / 2, \pi / 2\left[{ }^{3}\right.$ is the union of the six edges of $C$ which have no vertex $(-\pi / 2,-\pi / 2,-\pi / 2)$ or $(\pi / 2, \pi / 2, \pi / 2)$. We call this closed path the equator of $C$. The surface $\Sigma_{0}$ is a smooth 2-disk whose boundary is the equator of $C$.

The set $\mathcal{M}_{G}$ is the cone $\sum_{i=1}^{3} m_{i}^{2} \leq 2 \sum_{1 \leq i<j \leq 3} m_{i} m_{j}$. In particular, this closed set is not equal to $\mathcal{P}_{E}$.

\subsection{The eight figure}

Let us denote by $G_{8}$ the graph with one vertex and two edges (two loops). We have

$$
\delta_{G_{8}}\left(\theta_{1}, \theta_{2}\right)=\sin \frac{\theta_{1}}{2} \sin \frac{\theta_{2}}{2} \sin \frac{\theta_{1}+\theta_{2}}{2} .
$$

The set $Z_{G_{8}}$ is the union of the circles $\theta_{1}=0, \theta_{2}=0$ and $\theta_{1}+\theta_{2}=0$. The image by the Gauß map is the union of the three lines $m_{2}=0, m_{1}=0$ and $m_{1}=m_{2}$. The associated densities are up to global normalization $l_{1}, l_{2}, l_{1}+l_{2}$. 

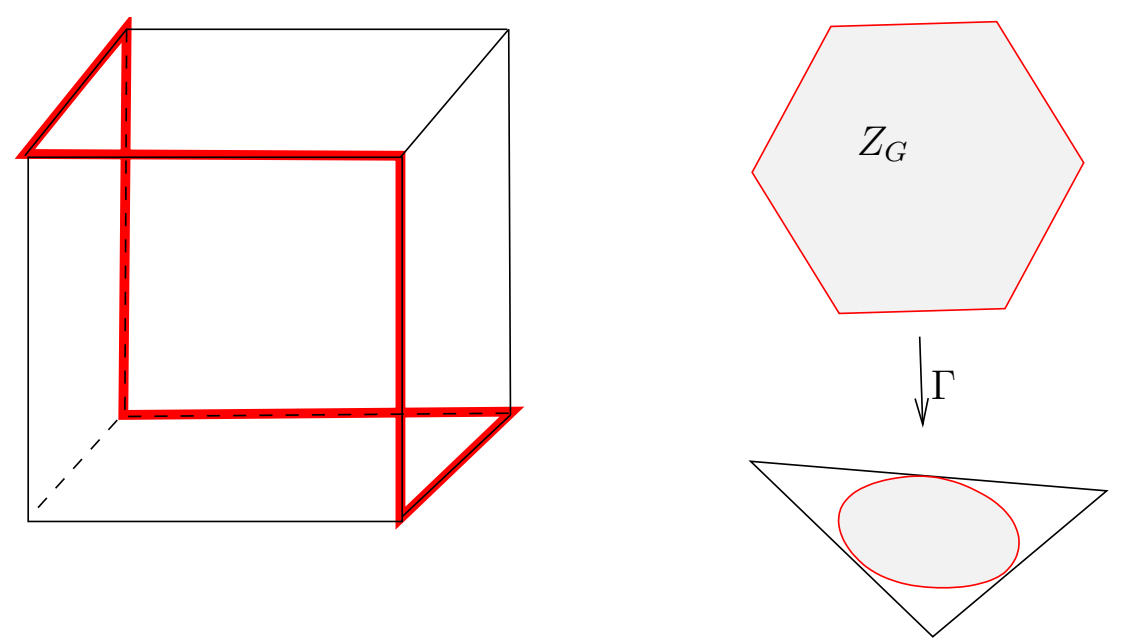

Figure 2: the surface $Z_{\text {star }}$ and the equator of the cube; the map $\Gamma$ from $Z_{\text {star }}$ to the semi-classical measures.

This implies that there exactly three semi-classical limits for an irrational length vector. The Liouville measure $\left|d t_{1}\right|+\left|d t_{2}\right|$ has relative density $\frac{1}{2}$. On this example we see that the set of semi-classical measure is not even connected and that the scars give measures which are extremal point of their convex hull.

\subsection{The cherry}

Let $G_{C}$ be the cherry graph consisting of one loop with an attached edge. Let us denote by $l_{1}$ the length of the loop and by $l_{2}$ the length of the edge. We get for $Z_{G_{C}}$ the equation:

$$
\sin \theta_{1} \sin \theta_{2}+2\left(1-\cos \theta_{1}\right) \cos \theta_{2}=0 .
$$

The set $Z_{G_{C}}$ is the union of the three circles

$$
\left\{\theta_{1}=0\right\} \cup\left\{\tan \theta_{2}+2 \tan \theta_{1} / 2=0\right\}
$$

The circle $\left\{\theta_{1}=0\right\}$ correspond to the scars localized on the loop ; the other part corresponds to a continuum of semi-classical measures $m_{1}\left|d t_{1}\right|+m_{2}\left|d t_{2}\right|$ with $m_{1} l_{1}+m_{2} l_{2}=1,1 \leq m_{2} / m_{1} \leq 4$.

\section{Limits of graphs and a new proof of Friedlan- der's Theorem}

From the characterization of the spectrum of $\Delta_{\vec{l}}$ as the set of $k^{2}$ so that $[k \vec{l}]$ belongs to $Z_{G}$, on can still define the spectrum of $\Delta_{\vec{l}}$ for $\vec{l}$ a real vector. In 
particular, one can take some lengths to be zero and the others $>0$. What is then the interpretation of this "spectrum"?

Let $G$ be a graph as before. Given a set of edges $X \subset E$, we introduce a new graph $G_{X}$ obtained by contracting all edges $e \in X$. From the point of view of Riemannian metrics, the graph $G_{X}$ can be interpreted as the graph $G$ where the lengths of the edges in $X$ vanish. We have the

Lemma 7.1 For any connected graph $G=(V, E)$ not homeomorphic to the circle or the interval, there exists a set $X \subset E$ so that $G_{X}$ is homeomorphic to the star graph with three edges, to $G_{8}$ or to $G_{C}$.

Let us denote by $b_{1}(G)$ the first Betti number of $|G|$, i.e. the dimension of the space of cycles of $|G|$.

The first case occur if $G$ is a tree not reduced to an interval, the second one if $b_{1}(G) \geq 2$ and the third one if $b_{1}(G)=1$ and $|G|$ is not a circle.

Let $\left(Z_{G}\right)_{X}$ be the intersection of $Z_{G}$ with the torus $\mathcal{T}_{X}=\left\{\theta_{e}=0, \forall e \in X\right\}$. We have clearly an identification of $\left(Z_{G}\right)_{X}$ with $Z_{G_{X}}$.

Any non singular point of $Z_{G_{X}}$ is in this way associated to a non singular point of $Z_{G}$. This gives an independent proof of Friedlander's result by using the fact that, for each of the previous reduced graphs, the manifold $Z_{G}$ admits non singular points.

\section{A The derivatives of the eigenvalues w.r. to the edge lengths}

This Lemma is contained in [Fr05]:

Lemma A.1 If $\lambda$ is a non degenerate eigenvalue of $\Delta_{\vec{l}}$ with a normalized eigenfunction $\phi\left(t_{e}\right)=a_{e} \cos k t_{e}+b_{e} \sin k t_{e}$ on the edge $e$, the derivative of $\lambda w . r$. to $l_{e}$ is given by

$$
\frac{\partial \lambda}{\partial l_{e}}=-\lambda\left(a_{e}^{2}+b_{e}^{2}\right)
$$

Proof.-

Let us put $\left.t=t_{e}, I=\right]-l_{e} / 2, l_{e} / 2[$ and choose a function $\psi \in$ $C_{o}^{\infty}(I, \mathbb{R})$ and the metric $g_{u}=\exp (4 u \psi(t))|d t|^{2}$ on the edge $e$ and independent of $u$ on the other edges. Then we have $d l_{e} / d u=2 \int_{I} \psi(t)|d t|$,

$$
\|\phi\|_{u}^{2}=\|\phi\|_{0}^{\prime 2}+\int_{I} e^{2 u \psi(t)} \phi^{2}(t)|d t|
$$

and the Dirichlet integral

$$
q_{u}(\phi)=q_{0}^{\prime}(\phi)+\int_{I} e^{-2 u \psi(t)} \phi^{\prime 2}(t)|d t|
$$


where ${ }_{0}^{\prime}$ denotes the integrals on the other edges which are independent of $u$. Let now take the $u$-derivative of the Rayleigh quotient $q_{u}(\phi) /\|\phi\|_{u}^{2}$ at $u=0$, we get

$$
d \lambda / d u=-2 \int_{I} \psi(t)\left(\phi^{\prime}(t)^{2}+\lambda \phi(t)^{2}\right)|d t|
$$

from which the result follows.

\section{B Appendix: the transition probabilities for a quantum graph}

We want to describe the way a wave arriving at a vertex of a quantum graph splits into several waves. Let us denote by $O$ the vertex of degree $d$ and by $e_{j}, j=$ $1, \cdots, d$ the $d$-edges arriving at $O$. Let us denote the arc-length coordinate $x_{j}$ along $e_{j}$ starting from 0 at $O$. Let us consider a function $f_{1}$ compactly supported in $] 0,+\infty\left[\right.$ and a wave $u(x, t)$ defined on $e_{1}$ by $u\left(x_{1}, t\right)=f_{1}\left(x_{1}+t\right)+g_{1}\left(x_{1}-t\right)$ and, on $e_{j}$ for $2 \leq j \leq d$, by $u\left(x_{j}, t\right)=g_{2}\left(x_{j}-t\right)$. From the Kirchhoff conditions, we get $f_{1}(t)+g_{1}(-t)=g_{2}(-t)$ and $f_{1}^{\prime}(t)+g_{1}^{\prime}(-t)+(d-1) g_{2}^{\prime}(-t)=0$. Integrating the second equation, we get $f_{1}(t)=g_{1}(-t)+(d-1) g_{2}(-t)$ (we see that the integration constant vanishes by putting $t=0)$. So that we get

$$
g_{1}(t)=\frac{2-d}{d} f_{1}(-t), g_{2}(t)=\frac{2}{d} f_{1}(-t) .
$$

The transition probabilities are the numbers

$$
p_{j, j}=\left(\frac{d-2}{d}\right)^{2}
$$

and for $i \neq j$,

$$
p_{i, j}=\left(\frac{2}{d}\right)^{2}
$$

The quantum graph is said classically ergodic if the following Markov process defined on the unit tangent bundle of the metric graph is ergodic: follow the edges with unit speed and arriving at a vertex use the transition probabilities defined before. We can assume that the graph has no vertex of degree two.

\section{Appendix: the case $\vec{l}=(1,1, \cdots, 1)$}

In this case the spectrum is given by the $k_{j}^{2}, k_{j} \geq 0$ so that $[k] \in Z_{G}$. Using Weyl asymptotics, the number of $k_{j}$ 's with multiplicity is $2 \# E$. On the other hand, 
we find from [Ni84, Ni87, Catt97], that the eigenvalues of $\Delta_{\overrightarrow{1}}$ are given in terms of the eigenvalues $\mu_{l}, l=1, \cdots, \# V$, of the weighted adjacency operator defined

$$
A_{G} f(i)=\frac{1}{d_{i}} \sum_{j \sim i} f(j) .
$$

The result is the following one for the $k_{j}$ :

- For each $\left|\mu_{l}\right|<1,2$ values of $k$ defined by $\cos k=\mu_{l}$.

- The value $k=0$ with multiplicity $1+b_{1}(G)$.

- The value $k=\pi$ with multiplicity $b_{1}(G)+1$ if $G$ is bipartite and $b_{1}(G)-1$ if-not.

At the end, we have, in the bipartite case $2 \# E=2(\# V-2)+2 b_{1}(G)+2$, and in the non-bipartite case $2 \# E=2(\# V-1)+2 b_{1}(G)$. Both formulas give the Euler formula for $G$ :

$$
\# V-\# E=1-b_{1}(G) \text {. }
$$

\section{Appendix: a Lemma on polynomial quasi- periodic functions}

Let $P$ be a non zero real valued polynomial of $2 N$ variables and consider, for $\alpha \in R^{N}$, the function

$$
f(t):=P\left(\cos t \alpha_{1}, \sin t \alpha_{1}, \cos t \alpha_{2}, \cdots\right) .
$$

We have the

Lemma D.1 The number $N(x)$ of zeroes (with multiplicity) of $f$ in the interval $[x, x+1]$ is uniformly bounded for $x \in \mathbb{R}$.

Proof.-

The function $f(t)$ extends to a bounded holomorphic function in the strip $-2<\Im t<2$. The set of functions $f_{x}(t)=f(t-x) /\|f\|_{L^{\infty}\left(\left[x_{1}, x+2\right] \times[-1,+1]\right)}$ is therefore a compact set of holomophic functions on $D=[-1,2] \times$ $[-1,+1]$. Let us assume that $N(x)$ is unbounded, we can choose a sequence $x_{j}$ so that $N\left(x_{j}\right) \rightarrow \infty$ and $f_{x_{j}}$ converges on $D$ to a non-zero holomorphic function $f_{\infty}$. For $j$ large enough, the number of zeroes $N\left(x_{j}\right)$ of $f_{x_{j}}$ on $[0,1]$ is less than the the (finite) number of zeroes of $f_{\infty}$ on $[-1,2]$. The contradiction follows. 


\section{References}

[Ba12] Ram Band. The Nodal Count $\{0,1,2,3, \ldots\}$ Implies The Graph is a Tree. Phil. Trans. of the Royal Society A372:20120504 (2014).

[BB13] Ram Band \& Gregory Berkolaiko. Universality of the momentum band density of periodic networks. Phys. Rev. Lett. 111:130404 (2013).

[BG00] Felipe Barra \& Pierre Gaspard, On the Level Spacing Distribution in Quantum Graphs, Journal of Statistical Physics 101:283-319 (2000).

[BG01] Felipe Barra \& Pierre Gaspard, Classical dynamics on graphs, Physical Review E 63:066215 (2001).

[BK13] Gregory Berkolaiko \& Peter Kuchment, Introduction to quantum graphs, Mathematical Surveys and Monographs (AMS), 186 (2013).

[BKW04] Gregory Berkolaiko, Jonathan Keating \& Brian Winn, No Quantum Ergodicity for Star Graphs. Commun. Math. Phys. 250:259-285 (2004).

[BW08] Gregory Berkolaiko \& Brian Winn, Relationship between scattering matrix and spectrum of quantum graphs. Transactions of the AMS 362:62616277 (2010).

[Catt97] Carla Cattaneo. The Spectrum of the Continuous Laplacian on a Graph. Monatshefte für Mathematik 124:215-235 (1997).

[CdV85] Yves Colin de Verdière, Ergodicité et fonctions propres du laplacien. Commun. Math. Phys. 102:497-502, (1985).

[Fr05] Leonid Friedlander, Genericity of simple eigenvalues for a metric graph, Israel J. Math., 146:149-156 (2005).

[GKF10] Sven Gnutzman, Jon P. Keating \& Fabien Piotet. Eigenfunction statistics on quantum graphs, Ann. Phys. 325:2595-2640 (2010).

[JSS13] Dmitry Jakobson, Yuri Safarov \& Alexander Strohmaier, The semiclassical theory of discontinuous systems and ray-splitting billiards (with an Appendix of Yves Colin de Verdière). American Journal of Maths (to appear) and ArXiv 1301.6783v1 (2013).

[KMW02] Jon P. Keating, Jens Marklof \& Brian Winn. Value Distribution of the Eigenfunctions and Spectral Determinants of Quantum Star Graphs. Commun. Math. Phys. 241:421-452 (2003).

[Ni84] Serge Nicaise, Some results on spectral theory over networks, applied to nerve impulse transmission, Orthogonal polynomials and applications (Barle-Duc, 1984), Lecture Notes in Math. (Springer), 1171:532-541 (1985). 
[Ni87] Serge Nicaise, Approche spectrale des problèmes de diffusion sur les réseaux, Séminaire de Théorie du Potentiel, Paris, No. 8, Lecture Notes in Math. (Springer), 1235:120-140 (1987).

[SK03] Holger Schanz \& Tsampikos Kottos. Scars on Quantum Networks Ignore the Lyapunov Exponent. Phys. Rev. Lett. 90:234101 (2003).

[Shn74] Alexander I. Shnirelman, Ergodic properties of eigenfunctions. Uspehi Mat. Nauk 29:181-182, (1974).

[Shn93] Alexander I. Shnirelman, On the asymptotic properties of eigenfunctions in the regions of chaotic motion. In V. Lazutkin KAM theory and semiclassical approximations to eigenfunctions. Ergebnisse der Mathematik und ihrer Grenzgebiete (3), 24. Springer-Verlag, Berlin, (1993].

[Zel87] Steve Zelditch, Uniform distribution of eigenfunctions on compact hyperbolic surfaces. Duke Math. J. 55:919-941 (1987). 